\title{
Ocorrência das doenças parasitárias em ovinos e caprinos no Distrito Federal, Brasil, durante 2003 a 2009
}

\author{
Occurrence of parasitic diseases in sheep and \\ goats in the District Federal, Brazil, during 2003 to 2009
}

\author{
Hélio Spindola de Ataíde ${ }^{1}$, Edison Rogerio Cansi ${ }^{2 *}$
}

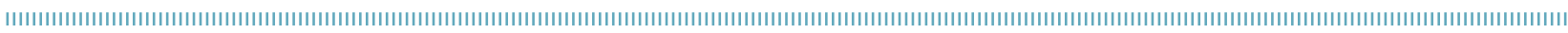

RESUMO: O parasitismo é um dos principais fatores limitantes na produçáo animal em geral. A ovinocultura e caprinocultura são afetadas por diversos parasitas que representam significativas perdas em carne, leite e lá da cultura. A introdução recente de ovinos e caprinos no Centro-Oeste brasileiro trouxe consigo novas enfermidades antes não relatadas na regiáo. $\mathrm{O}$ objetivo deste estudo é descrever a ocorrência dos principais parasitas que afetam os caprinos e ovinos no Distrito Federal e região do entorno e os fatores predisponentes destas enfermidades ao rebanho local, revisando os casos obtidos pela necropsia dos animais no período de 2003 a 2009. Nos meses de outubro de 2009 a julho de 2010 foram revistos os laudos de necropsia da regiáo, em retrospectiva dos anos de 2003 a 2009, à procura das casuísticas por doenças parasitárias. Verificou-se a ocorrência de endoparasitoses e ectoparasitoses em pequenos ruminantes. Os principais parasitas encontrados foram o Haemonchus contortus e Oestrus ovis nas duas culturas, porém Oesophagostomun sp. ocorreu apenas em ovinos. Encontrou-se maior ocorrência de Haemonchose na estaçáo chuvosa do Cerrado. Este é o primeiro trabalho epidemiológico descritivo sobre parasitismo em cabras e ovelhas na regiáo.

PALAVRAS-CHAVE: ectoparasitas; endoparasitas; epidemiologia; ovinocultura; caprinocultura.

\begin{abstract}
Parasitism is a major factor limiting livestock production in general. The goat and sheep industry are affected by many parasites representing significant losses in meat, milk and wool culture. The recent introduction of sheep and goats in the Brazilian Midwest region brought with them new diseases not previously reported in the region. The aim of this study is to describe the occurrence of major parasites, affecting sheep and goats in the Federal District and surrounding areas, and predisposing factors of these diseases to local herd reviewing cases obtained by necropsy of animals in the period 2003 to 2009. From October 2009 to July 2010 were reviewed the necropsy reports of the region in retrospect the years 2003 to 2009 looking for the demand for case studies of parasitic diseases. It was found to occur of ectoparasitosis and endoparasitosis in small ruminants. The main parasites found were Haemonchus contortus and Oestrus ovis in both cultures, however Oesophagostomun sp. occurred only in sheep. There was a higher occurrence of Haemonchose in the rainy season in the Cerrado. This is the first epidemiological research of parasitism in goats and sheep in the region.
\end{abstract}

KEYWORDS: ectoparasitis; endoparasitis; epidemiology; ovinoculture; goat culture. 
O parasitismo é a interação ecológica entre indivíduos de diferentes espécies nas quais hospedeiros e parasitas mantêm uma associaçáo unilateral, íntima e direta com certo grau de dependência metabólica por parte dos parasitas (ForTes, 1997; Poulin, 2007). Os resultantes destas relaçôes desarmônicas representam danos significativos aos hospedeiros (Otranto, 2001; TaYlor et al., 2007). Na produção animal, o parasitismo é um dos principais limitantes, impondo diversos desafios no combate às enfermidades por eles geradas (Waller, 1999; Molento, 2004). A ovinocultura e a caprinocultura foram as primeiras culturas com uso de animais praticadas pelo homem (Clutton-Brock, 1999; Mazoyer; ROUdART, 2001). Elas sempre foram limitadas por diversos parasitas, obrigando os produtores a buscarem inovaçôes constantes em uma corrida evolutiva de resistência deles e introduçáo de novas tecnologias em seu combate (MотA et al., 2003; Molento, 2004).

Uma grande diversidade de parasitas afeta ovelhas e cabras, sendo os principais os endoparasitas Haemonchus contortus (RAmos et al., 2004; Cavalcante et al., 2009), Trichostrongylus axei, Oesophagostomum sp., Strongyloides sp. (Amarante et al., 2004; Ramos et al., 2004), diversas espécies de Cooperia e Ostertagia, Fasciola hepatica (Pugh, 2002; Ramos et al., 2004; Cavalcante et al., 2009; Smith; Sherman, 2009), Moniezia sp. e Taenia hydatigena (Christodoulopoulos et al., 2008), além dos protozoários Eimeria sp., Cryptosporidium sp., Babesia sp. e Toxoplasma gondii (Pugh, 2002; Broughan; Wall, 2007; Dubey, 2009; Cavalcante et al., 2009). As doenças ectoparasitárias de destaque nestes animais domésticos são a pediculose, sarnas, míases, principalmente a nasal por Oestrus ovis, e as enfermidades resultantes da ação vetorial pelos carrapatos (FouRIE; Horak, 2000; Madeira et al., 2000; ColWell et al., 2006; BisDorfF; WALL, 2008). Diversas espécies parasitárias são frequentemente encontradas em ovinos e caprinos na criaçáo em regiôes tropicais, muitas vezes com alta patogenicidade, ocasionando perdas econômicas significativas e até mesmo o óbito de seus hospedeiros, exemplificada pelos diversos casos oriundos da ação por $H$. contortus (VATTA et al., 2001; RAmos et al., 2004).

No Centro-Oeste brasileiro, com destaque para o estado de Goiás e o Distrito Federal, a ovinocultura e a caprinocultura são culturas recentes, introduzidas na década de 1980 com representativos incrementos dos rebanhos a cada ano (Brasil, 2008). A chegada dos animais trouxe consigo novos desafios sanitários, incluindo as doenças parasitárias. As práticas de manejo em geral na região correspondem às adaptaçôes dos conhecimentos adquiridos para a prática em outras localidades do Brasil e do mundo, já tradicionais na criação (Vieira, 2005; Pugh, 2002; Cavalcante et al., 2009).

O objetivo deste estudo é descrever a ocorrência e os fatores predisponentes das endoparasitoses e ectoparasitoses obtidas nas necropsias de ovinos e caprinos produzidos no Distrito Federal e entorno (regiáo dos estados de Goiás, Minas Gerais e Bahia) durante os anos de 2003 a 2009.

Entre outubro de 2009 e julho de 2010 foram revisados os laudos de necropsia do Laboratório de Patologia Veterinária da Universidade de Brasília (UnB) em retrospectiva dos anos 2003 a 2009, verificando a ocorrência de parasitoses (Endo- e Ectoparasitas) em ovinos e caprinos no Distrito Federal e região do entorno e relatando as espécies ou gêneros identificados. O estudo revisional é um trabalho descritivo e apresenta a estatística descritiva da ocorrência das parasitoses em ovinos e caprinos. Este é o primeiro trabalho epidemiológico sobre parasitismo em cabras e ovelhas da região.

Entre os anos de 2003 a 2009, 365 ovinos e 83 caprinos foram necropsiados. Do total, 83 (27,7\%) ovelhas estavam parasitadas por uma ou mais espécie de parasitas comum à produção, e 18 (25,7\%) cabras apresentavam um ou mais parasitismo. Multiparasitismo esteve presente em $19(22,9 \%)$ dos ovinos e em 4 (22,2\%) dos caprinos. A ocorrência da associação entre endoparasitas e ectoparasitas foi de $11,1 \%(n=2)$ em cabras e $12,0 \%(n=10)$ em ovelhas. Os parasitas com maior ocorrência diagnosticadas em ambas as culturas foram Haemonchus contortus e Oestrus ovis (miíase nasal). Na ovinocultura regional, verificou-se alta ocorrência de Oesophagostomum sp. (Tabela 1).

As doenças parasitárias pediculose e miíase cutânea não foram identificadas ao nível de espécies, porém constatou-se

Tabela 1. Frequência dos parasitas em ovinos e caprinos necropsiados pelo Laboratório de Patologia Veterinária da Universidade de Brasília nos anos de 2003 a 2009.

\begin{tabular}{|c|c|c|c|c|}
\hline \multirow{2}{*}{ Parasitas } & \multicolumn{2}{|c|}{ Ovinos } & \multicolumn{2}{|c|}{ Caprinos } \\
\hline & n & $\%$ & $\mathbf{n}$ & $\%$ \\
\hline \multicolumn{5}{|l|}{ Ectoparasitismo } \\
\hline Ixodidae & 3 & 2,9 & 1 & 4,5 \\
\hline Oestrus ovis & 20 & 19,8 & 3 & 13,7 \\
\hline Miíase cutânea & 3 & 2,9 & 0 & 0,0 \\
\hline Pediculose & 0 & 0,0 & 1 & 4,5 \\
\hline \multicolumn{5}{|c|}{ Nematoides Gastrointestinais } \\
\hline Haemonchus contortus & 45 & 44,4 & 9 & 41,0 \\
\hline Oesophagostomum sp. & 14 & 13,8 & 0 & 0,0 \\
\hline Trichuris sp. & 1 & 0,9 & 0 & 0,0 \\
\hline Trichostrongylus axei & 1 & 0,9 & 1 & 4,5 \\
\hline \multicolumn{5}{|l|}{ Cestodas } \\
\hline Taenia hydatigena & 3 & 2,9 & 2 & 9,1 \\
\hline Moniezia sp. & 4 & 3,9 & 2 & 9,1 \\
\hline \multicolumn{5}{|l|}{ Trematoda } \\
\hline Fasciola hepatica & 1 & 0,9 & 1 & 4,5 \\
\hline \multicolumn{5}{|l|}{ Protozoários } \\
\hline Eimeria sp. & 5 & 4,9 & 2 & 9,1 \\
\hline Toxoplasma gondii & 1 & 0,9 & 0 & 0,0 \\
\hline Sarcocystis sp. & 1 & 0,9 & 0 & 0,0 \\
\hline TOTAL & 102 & 100,0 & 22 & 100,0 \\
\hline
\end{tabular}


o relato das enfermidades. Observou-se nos relatos das fichas, descrição de animais parasitados por espécies de cestodas, trematodas (Fasciola hepatica), protozoários e outros nematodas gastrintestinais.

Constatou-se que a haemoncose apresentou maior ocorrência nos meses de abril $(n=14)$ e março $(n=6)$ (Fig. 1), período correspondente à estação chuvosa no cerrado brasileiro para os ovinos, e no ano de $2008(\mathrm{n}=3)$ e mês de março $(\mathrm{n}=2)$ para caprinos.

A alta ocorrência do parasitismo nas necropsias de ovinos em caprinos no Distrito Federal e entorno corrobora os trabalhos realizados em outras regióes do Brasil (Arosemena et al., 1999; Ramos et al., 2004). Observou-se que as principais formas de parasitismo com maior casuística foi gastrenterite parasitária em pequenos ruminantes, situação já evidenciada em outros estudos nas regiōes Sul, Nordeste e Sudeste do País (Amarante et al., 1996; Arosemena et al., 1999; Ramos et al., 2004; CavalCANTE et al., 2009).

Verificou-se alta ocorrência de Oestrus ovis (Diptera: Oestridae). Esta mosca é causadora das miíases nasais em ovelhas e cabras, responsável por perdas significativas na produção nas regióes Sul e Sudeste do Brasil. Sua representativa ocorrência indica expansão do parasitismo no país, acompanhando o desenvolvimento da cultura de pequenos ruminantes (Guimaráes; Papavero, 1999; Colwell et al., 2006). O presente estudo e outros preparados pelos autores desta descrição epidemiológica são os primeiros relatos da expansão da oestrose no Distrito Federal e entorno (principalmente Goiás).

O número de necropsias de ovinos e caprinos produzidas no Distrito Federal ainda é expressivamente pequeno quando comparado aos demais animais de produção, principalmente bovinos. Este fato está relacionado ao pequeno número do rebanho ainda presente na região, devido à recente introdução destas culturas (BRASIL, 2008).

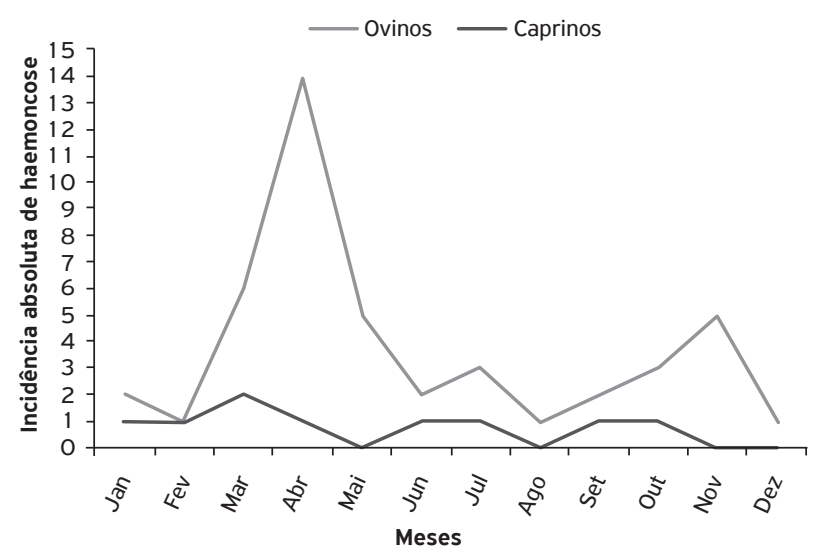

Figura 1. Ocorrência de Haemoncose em Ovinos e Caprinos no Distrito Federal, Brasil, durante 2003 a 2004.
O relato dos nematodas Haemonchus contortus, Trichostrongylus axei e Oesophagostomum sp. nos laudos retoma a importância destes parasitas como limitantes produtivos e a necessidade de manejo adequado para combatê-los também no Centro-Oeste brasileiro (Amarante et al., 2004).

Os endoparasitas foram evidenciados como importantes causadores de enfermidades na ovinocultura e caprinocultura em outros estudos nas regióes Sudeste (Amarante et al., 2004; Chagas et al., 2008), Nordeste (Arosemena et al., 1999) e Sul (Ramos et al., 2004) do País, sendo o H. contortus o principal nematoda causador de perdas em toda a produçáo destes animais no Brasil e no mundo; sendo assim o parasita-chave na limitação da ovinocultura (CAVAlCANTE et al., 2009). H. contortus foi quem apresentou maior incidência absoluta nos animais necropsiados durante 2003 a 2009 no Distrito Federal, evidenciando sua expressiva ação também no Centro-Oeste brasileiro. A diferença na incidência ano a ano é explicada pelo aumento no número de animais, principalmente ovelhas, na localidade a partir de 2007, valendo destacar que a caprinocultura ainda é uma criação incipiente em Brasília e entorno (Brasil, 2008).

Os meses de março a abril, quando há ocorrência de haemoncose, coincidem com a estação das chuvas no cerrado, no entanto, é possível encontrar o nematoda o ano inteiro em ovinos. Estas informaçôes são semelhantes às encontradas no Sudeste (CAvalcante et al., 2009), porém, nesta regiáo, a incidência maior é no período com temperatura mas baixa (Amarante et al., 2004; Chagas et al., 2008). A informação é coerente com ciclo da espécie $H$. contortus, pois este parasita necessita alta umidade atmosférica e temperatura ideal de $23^{\circ} \mathrm{C}$ para o seu desenvolvimento no meio ambiente (ForTEs, 1997; RAMOs et al., 2004).

Este é o primeiro trabalho descritivo das doenças parasitárias em ovinos e caprinos no Distrito Federal do Brasil e áreas do entorno e tem como objetivo alertar e instigar os pesquisadores locais para a nova situação sanitária desta recente cultura de produção animal introduzida.

\section{AGRADECIMENTOS}

Agradecemos ao Conselho Nacional de Desenvolvimento Científico e Tecnológico (CNPq) pela bolsa de doutoramento de Edison Rogerio Cansi, o Programa de Pós-Graduaçáo em Biologia Animal do Instituto de Ciências Biológicas da Universidade de Brasília pelo suporte acadêmico e científico, e o suporte técnico do Laboratório de Patologia Animal da Universidade de Brasília. 


\section{REFERÊNCIAS}

AMARANTE, A.F.T.; BRICARELLO, P.A.; ROCHA, R.A.. Resistance of Santa Ines, Suffolk and lle de France lambs to naturally acquired gastrointestinal nematode infections. Veterinary Parasitology, v.120, n.1, p.91-106, 2004.

AMARANTE, A.F.T.; PADOVANI, C.R.; BARBOSA, M.A. Contaminação da pastagem por larvas infectantes de nematódeos gastrintestinais parasitas de bovinos e ovinos em Botucatu - SP. Revista Brasileira de Parasitologia Veterinária, v.5, p.65-73, 1996

AROSEMENA, N.A.E.; BEVILAQUA, C.M.L.; MELO, A.C.F.L.; GIRÃO, M.D. Seasonal variations of gastrointestinal nematodes in sheep and goats from semi-arid area in Brazil. Revue de Médicine Véterinaire, v.150, p.873-876, 1999.

BISDORFF, B.; WALL, R. Control and management of sheep mange and pediculosis in Great Britain. Veterinary Parasitology, v.155, n.1-2, p.120-126, 2008.

BRASIL. Instituto Brasileiro de Geografia e Estatística. Produção da pecuária municipal - 2007. Rio de Janeiro: IBGE, 2008. 62p.

BROUGHAN, J.M.; WALL, R. Faecal soiling and gastrointestinal helminth infection in lambs. International Journal of Parasitology, v.37, n. 11 , p.1255-1268, 2007.

CAVALCANTE, A.C.R.; VIEIRA, L.S.; CHAGAS, A.C.S.; MOLENTO, M.B. (Eds.). Doenças parasitárias de caprinos e ovinos: epidemiologia e controle. Brasília: Embrapa Informação Tecnológica, 2009. 603p.

CHAGAS, A.C.S.; OLIVEIRA M.C.S.; ESTEVES, S.N.; OLIVEIRA, H.N.; GIGLIOTI, R.; GIGLIOTI, C.; FERREZINI, J.; SCHIAVONE, D.C. Parasitismo por nematóides gastrintestinais em matrizes e cordeiros criados em São Carlos, São Paulo. Revista Brasileira de Parasitologia Veterinária, v.17, p.126-132, 2008.

CHRISTODOULOPOULOS, G.; THEODOROPOULOS G.; PETRAKOS, G. Epidemiological survey of cestode-larva disease in Greek sheep flocks. Veterinary Parasitology, v.153, n.3-4, p.368-373, 2008.

CLUTTON-BROCK, J. A natural history of domesticated mammals. Cambridge: Cambridge University Press, 1999. 248p.

COLWELL, D.D.; HALL, M.J.R.; SCHOLL, P.J. The oestrid flies: biology, host-parasite relationships, impact and management. Oxfordshire: $\mathrm{CABI}, 2006.359 \mathrm{p}$.

DUBEY, J.P. Toxoplasmosis in sheep - the last 20 years. Veterinary Parasitology, v.163, n.1-2, p.1-14, 2009.

FORTES, E. Parasitologia veterinária. São Paulo: Ícone, 1997.607p

FOURIE, L.J.; HORAK, I.G. Status of Dorper sheep as hosts of ectoparasites. Small Ruminant Research, v.36, n.2, p.159164, 2000.
GUIMARÃES, J.H.; PAPAVERO, N. Myiasis in man and animals in the Neotropical region. São Paulo: FAPESP/Editora Plêiade, 1999. 308p.

MADEIRA, N.G.; AMARANTE, A.F.; PADOVANI, C.R. Diversity of ectoparasites in sheep flocks in São Paulo, Brazil. Tropical Animal Health and Production, v.32, n.4, p.225-232, 2000.

MAZOYER, M.; ROUDART, L. História das agriculturas do mundo: do Neolítico à crise contemporânea. Lisboa: Instituto Piaget. 2001, 567p.

MOLENTO, M.B. Resistência de helmintos em ovinos e caprinos. Revista Brasileira de Parasitologia Veterinária, v.13, p.82-87, 2004.

MOTA, M.A.; CAMPOS, A.K.; ARAÚJO, J.V. Controle biológico de helmintos parasitos de animais: estágio atual e perspectivas futuras. Pesquisa Veterinária Brasileira, v.3, n.3, p.93-100, 2003.

OTRANTO, D. The immunology of myiasis: parasite survival and host defense strategies. Trends in Parasitology, v.17, n.4, p.176-182, 2001

PAPAVERO, N. The world oestridae, mammals and continental drift. Haia: Dr. W. Juhk Publishers, 1976. 240p.

POULIN, R. Evolutionary ecology of parasites. Princeton: Princeton University Press, 2007. 332p.

PUGH, D.G. Sheep and goat medicine. Philadelphia: Elsevier Health Sciences. 2002. 468p.

RAMOS, C.I.; BELLATO, V.; SOUZA, A.P.; AVILA, V.S.; COUTINHO, G.C.; DALAGNOL, C.A. Epidemiologia das helmintoses gastrintestinais de ovinos no Planalto Catarinense. Ciência Rural, v.34, n.6, p.1889-1895, 2004.

SMITH, M.C.; SHERMAN, D.M. Goat medicine. Ames: WilleyBlackwell, 2009. $871 \mathrm{p}$.

TAYLOR, M.A.; COOP, R.L.; WALL, R.L. Veterinary parasitology. Philadelphia: Willey-Blackwell, 2007. 600p.

VATTA, A.F.; LETTY, B.A.; VAN DER LINDE, M.J.; VAN WIJK, E.F.; HANSEN, J.W.; KRECEK, R.C. Testing for clinical anaemia caused by Haemonchus spp. in goats farmed under resource-poor conditions in South Africa using an eye colour chart developed for sheep. Veterinary Parasitology, v.99, n.1, p.1-14, 2001.

VIEIRA, L.S. Endoparasitoses gastrintestinais em caprinos $e$ ovinos. Sobral: Embrapa Caprinos, 2005. 32p.

WALLER, P.J. International approaches to the concept of integrated control of nematode parasites of livestock. International Journal for Parasitology, v.29, n.1, p.155164, 1999. 